\title{
Dynamik und Regelung - ein Forschungsprojekt der Linzer Mechatronik
}

Mechatronik, eine junge Ingenieurswissenschaft, ist inzwischen zu einem etablierten Wissensgebiet geworden, das fest in der Industrie und an den Universitäten verankert ist. Bereits mehrmals waren der Mechatronik Schwerpunkthefte der e\&i gewidmet; so wurde z.B. im Heft 1/2001 erstmals die Tätigkeit der Mechatronikgruppe in Linz vorgestellt.

In Linz wurde im Studienjahr 1990/1991 das weltweit erste Vollstudium der Mechatronik begonnen, das sich inzwischen zu einer stark nachgefragten akademischen Ausbildung entwickelt hat, die den industriellen und wissenschaftlichen Anforderungen des 21. Jahrhunderts Rechnung trägt. Zur Optimierung des Wissenstransfers zwischen Universität und Industrie wurde im Jahre $2001 \mathrm{im}$ Rahmen des K+-Programms des Bundesministeriums für Bildung, Wissenschaft und Kultur, vertreten durch die Technologie Impulse Gesellschaft, das Linzer Kompetenzzentrum für Mechatronik (Linz Center of Competence in Mechatronics) gegründet, das sich seither sehr erfolgreich der wissenschaftlichen Zusammenarbeit von Industrie und Universität widmet. Neben diversen industriellen Kooperationen der Linzer Mechatronikinstitute trägt auch das 1999 errichtete Christian Doppler Labor „Automatisierung mechatronischer Systeme der Stahlindustrie" zur Zusammenarbeit mit der Industrie, insbesondere in der grundlagenorientierten industriellen Forschung, bei.

Die Dynamik und Regelung mechatronischer Systeme, besonders solcher aus der Mechanik, ist einer der Forschungsschwerpunkte in Linz, dem sich junge Forscher aus dem Kompetenzzentrum in Zusammenarbeit mit der Universität und dem CD-Labor widmen. Dieses Heft soll nun einen Überblick über die laufenden Arbeiten bieten, wobei sich die Beiträge in zwei Gruppen zusammenfassen lassen. Die erste Gruppe befasst sich mit Problemen aus der Robotik und der angewandten Regelungstheorie. So behandelt der Beitrag über "Vielgliedrige elastische Roboter" die Modellierung von komplexen Manipulatoren, bei denen die elastischen Deformationen infolge von Leichtbau und hoher Dynamik nicht mehr vernachlässigbar sind. Neue Wege der Modellbildung mechatronischer Systeme, die bereits auf den Entwurf eines geregelten Systems zugeschnitten sind, werden im Aufsatz über „Das Tor-Konzept für die Modellbildung und Regelung komplexer dynamischer Systeme" beschrieben. Hier wird eine passivitätsbasierte Störregelung mit einer flachheitsbasierten Führungsregelung kombiniert. Letztere kommt auch beim Beitrag über „Flachheitsbasierte Folgeregelung des Labormodells Ball on the Wheel" zum Einsatz.

Die zweite Gruppe der Beiträge dieses Heftes ist auf die Modellierung von mechatronischen Systemen mit komplexer Dynamik fokussiert. So behandelt der Beitrag über die „Dynamik geregelter flexibler Mehrkörpersysteme unter Berücksichtigung hydraulischer Aktorik" Methoden zur systematischen Simulation von Maschinen und diskutiert numerische Integratoren für DAE-Systeme. Dass Stoßdämpfer eine sehr komplexe Dyna-

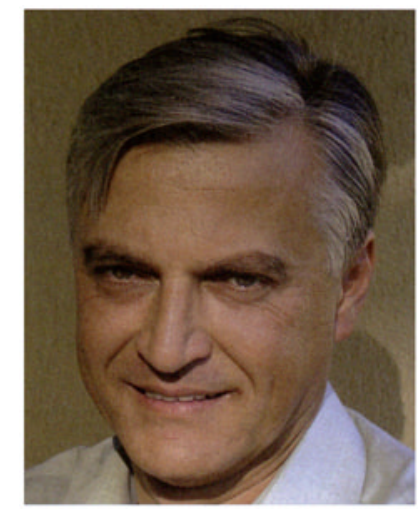

O. Univ.-Prof. Dipl.-Ing. Dr. Kurt Schlacher

mik aufweisen können, zeigt der Aufsatz über eine „Versuchsgestützte Modellierung von Kfz-Stoßdämpfern für die dynamische Mehrkörpersimulation", der die mathematische Modellbildung auf Grundlage physikalischer Prinzipien mit aussagekräftigen Laborversuchen verbindet. Abkantautomaten sind hoch automatisierte Maschinen zur Herstellung aufwendig geformter Profile aus ebenen Blechstücken. Wie Mechatronik zur Optimierung solcher Maschinen beitragen kann, ist dem Beitrag über die „Rechnergestützte nichtlineare Modellierung von Abkantautomaten" zu entnehmen. Ebenfalls sehr umfangreiche Maschinen, nämlich Walzgerüste, werden im Aufsatz über „Mechatronische Modellierung und Simulation der Dynamik von Walzanlagen" behandelt. Hier trägt geschickte Modularisierung zur Beherrschung hoch komplexer Simulationsmodelle bei. Hydraulische Verbraucher, die pulsartige Volumenströme erzeugen, können Druckschwankungen in der Versorgung auslösen. Eine systematische Untersuchung dieses Phänomens findet der Leser im Beitrag „Zur Dynamik von hydraulischen Common RailSystemen".

Mechatronik als Wissenschaft ist ja inzwischen zu einem weltweit wohl etablierten Gebiet geworden, das Grundlagenforschung mit angewandter Forschung auf integrative Weise verbindet. Die Beiträge dieses Heftes sollen dem Leser einen Einblick in die überaus lebendige Entwicklung der Mechatronik anhand des Forschungsschwerpunkts „Dynamik und Regelung mechanischer Systeme" der Linzer Mechatronikgruppe geben. Zusätzlich zur wissenschaftlichen Information soll das Heft dem Leser aber auch das nötige Vergnügen bieten, das für jede kreative wissenschaftliche Tätigkeit unentbehrlich ist

\section{O. Univ.-Prof. Dipl.-Ing. Dr. Kurt Schlacher}

Johannes-Kepler-Universität Linz

Institut für Regelungstechnik und Prozessautomatisierung und Christian Doppler-Laboratorium für Automatisierung mechatronischer Systeme der Stahlindustrie 